\title{
System-Structure Coupling Dynamic Analysis of Planetary Gears
}

\author{
Haiwei Wang, ${ }^{1}$ Tao Zhang, ${ }^{1,2}$ Geng Liu, ${ }^{1}$ and Liyan $\mathrm{Wu}^{1}$ \\ ${ }^{1}$ Shaanxi Engineering Laboratory for Transmissions and Controls, Northwestern Polytechnical University, Xian 710072, China \\ ${ }^{2}$ China Academy of Launch Vehicle Technology, R\&D Center, Beijing 100076, China \\ Correspondence should be addressed to Haiwei Wang; whw@nwpu.edu.cn
}

Received 26 December 2014; Accepted 21 May 2015

Academic Editor: Alessandro Gasparetto

Copyright (C) 2015 Haiwei Wang et al. This is an open access article distributed under the Creative Commons Attribution License, which permits unrestricted use, distribution, and reproduction in any medium, provided the original work is properly cited.

\begin{abstract}
This paper presents a novel general system-structure coupling dynamic analysis procedure to comprehensively analyze the dynamic performance of planetary gears. The novel coupling dynamic analysis takes dynamic loads of gears as excitations for structure dynamic analysis. Considering the time-varying mesh stiffness of gears, torsional stiffness of carrier and support stiffness of bearings, the system dynamic model of planetary gears is built by using lumped parameter method. Vibration modes and natural frequencies of planetary gears are investigated through modal analysis. Furthermore, system dynamic response is analyzed under various working conditions. Equations of structure dynamic analysis based on finite element method (FEM) are developed, and their solving method is put forward. Dynamic loads obtained from system dynamic analysis are forced on the ring gear to analyze its structure dynamic response. In every analysis step, if dynamic performance criteria are not satisfied, the planetary gears model should be redesigned according to dynamic analysis results.
\end{abstract}

\section{Introduction}

Planetary gears are important types of mechanical transmissions, which are typically composed of a sun gear, several planet gears, a ring gear, and a carrier. In comparison with parallel shaft trains, planetary gears offer many advantages such as higher speed reductions, higher power density, increased efficiency, and stronger stability [1]. They are widely used in aircrafts, ships, automobiles, wind turbines, and a variety of other applications. However, the vibration generated in planetary gears induces the noise of system and even influences the reliability of the whole machine. For example, cabin noise exceeding $100 \mathrm{~dB}$ is directly traceable to the planetary gears in some helicopters [2].

Analytical dynamics models of planetary gears are classified in three categories, namely, lumped parameter model, distributed mass model, and rigid-flexible coupling model. Lumped parameter model is the earliest and the most mature modeling method for planetary gears dynamics analyses. Every motion component is simplified as a mass point.
Motion components are interconnected by springs which represent mesh teeth and support bearings. Therefore, planetary gears are simplified as a spring-mass system, on which second-order differential equations are set up. According to the number of degree of freedom, the lumped parameter models can be further distinguished as purely rotational dynamics model $[3,4]$, rotational-transverse coupling dynamic model $[1,5]$, or rotational-transverse-shaft coupling dynamic model $[6,7]$. In distributed mass model, actions between gear teeth are simplified by using FE-contact model $[8,9]$. Time-varying mesh stiffness computed in this model is more accurate because of the introduction of simulation process. In rigid-flexible coupling model, some components, such as ring gear, are assumed as flexible bodies considering their small stiffness, while others are assumed as rigid bodies $[10,11]$. Abousleiman and Velex [12] developed a hybrid finite element/lumped parameter model based on a threedimensional finite element model of ring gear. Ericson and Parker [13] reported that the appearance of the natural frequencies of planetary gears tends to gather in to clusters. 
Three natural frequency clusters in relatively high frequencies region were recorded. Every natural frequency cluster includes a rotational vibration mode, a transverse vibration mode, and a planet vibration mode. Parker and $\mathrm{Wu}[14]$ analytically investigated the parametric instability of planetary gear system containing elastic ring gear based on a hybrid continuous-discrete model. Vibration mode properties of planetary gears are studied [15-17], and a famous feature indicated that vibration modes have obvious different motion forms. The vibration modes are classified in two categories, namely, distinct and degenerate mode [15]. Large numbers of investigations have been made in the previous research to discover the vibration mechanism of planetary gears; however, there are no effective methods to reduce both system and structure vibration of planetary gears.

Based on lumped parameter model and finite element method (FEM), a novel system-structure coupling dynamic analysis method of planetary gears is proposed. System dynamics and structure dynamics are coupled together through the dynamic load transfer. The analysis results demonstrate that the proposed coupling dynamic analysis method is a convenient approach to analyze the dynamic characteristics. The system and structure dynamic performances are obtained to guide the design of planetary gears simultaneously.

\section{System-Structure Coupling Dynamic Analysis Procedure of Planetary Gears}

2.1. General Procedure of System-Structure Coupling Dynamic Analysis. In planetary gears dynamic vibration, there are many excitations and influence factors, which include not only system parameters but also structure parameters. If the system and structure influence factors are considered at the same time, more accurate dynamic characteristics will be revealed. So a general procedure of system-structure coupling dynamic analysis is proposed, whose main contents are illustrated in Figure 1.

The proposed analysis procedure consists of four steps, which are stiffness and inertia computation based on analytical formulas, planetary gears modal analysis, system dynamic response analysis, and structure dynamic response analysis of gears based on FEM. Input data for those activities are gears model (including sun gear, planet gear, and ring gear), carrier model, support stiffness, and working condition. Output data generated are system dynamic performances (frequencies and dynamic loads) and structure dynamic stresses. Upon completion of every step, analysis results will be used to determine whether the planetary gears design needs to be improved or not.

2.2. Coupling Method of System Dynamics and Structure Dynamics. The connection of system dynamics with structure dynamics is the interaction forces between components. For solving the coupling problem, we propose a transfer load method. After analyzing system dynamic response of
TABLE 1: Basic design parameters of the example planetary gears.

\begin{tabular}{lccc}
\hline Parameter & Sun gear & Planet gear & Ring gear \\
\hline Number of teeth & 35 & 37 & 110 \\
Module, mm & 4.33 & 4.33 & 4.33 \\
Pressure angle, deg & 20 & 20 & 20 \\
Number of planet gears & - & 5 & - \\
Face width, mm & 58 & 58 & 58 \\
\hline
\end{tabular}

planetary gears, the dynamic loads on every gear can be obtained. Then the dynamic loads are applied on a 3D gear structure to carry out structure dynamic response analysis based on FEM.

2.3. Basic Parameters of Planetary Gears Model. An example planetary gears system is chosen to represent a helicopter gear set. The ring gear is held stationary and the other two central members are input and output, respectively. The basic design parameters of the example planetary gears are listed in Table 1 .

\section{System Dynamic Analysis of Planetary Gears}

3.1. System Dynamic Model of Planetary Gears. Because the axial influence of the planetary gears can be ignored, both rotational and translational motions of the planetary gears are considered in this paper. Therefore, each gear has three degrees of freedom, including two planar translations and one rotation. The system dynamic model of the planetary gears is illustrated in Figure 2, where the subscripts $S, C$, $R$, and $P$, respectively, represent the sun gear, the carrier, the ring gear, and the planet gears. The number of the planet gears is $N . u_{j}(j=S, C, R, P 1, P 2, \ldots, P N)$ are the rotational motions. $v_{i}, \xi_{i}(i=1,2, \ldots, N)$ are the radial and circumferential translational motions of the planet gears, respectively. $x_{S}, x_{C}, x_{R}$ and $y_{S}, y_{C}, y_{R}$ are the two planar translational motions, respectively. $k_{S u}, k_{C u}, k_{R u}$ are the circumferential support stiffness. $k_{C x}, k_{R x}$ and $k_{C y}, k_{R y}$ are the two translational support stiffness, respectively. Both the two translational support stiffness of the sun gear are $k_{S}$. Both the two translational support stiffness of each planet gear are $k_{P}$. $k_{S i}, k_{R i}(i=1,2, \ldots, N)$ are the external and internal mesh stiffness of planet $i(i=1,2, \ldots, N)$ with the sun gear and the ring gear, respectively. $J_{j}, m_{j}(j=S, C, R, P)$ are the rotational inertias and the masses, respectively. $\varphi_{i}$ is the location angle of planet gear $i$ in the global coordinate system. $\alpha$ is the pressure angle of each gear.

According to Newton second law, the following motion equations of the planetary gears are set up as in (1). The dampings in the system model are neglected. Total number of the degree of freedom of the system dynamic model is $3 \times(N+3)$. Consider 


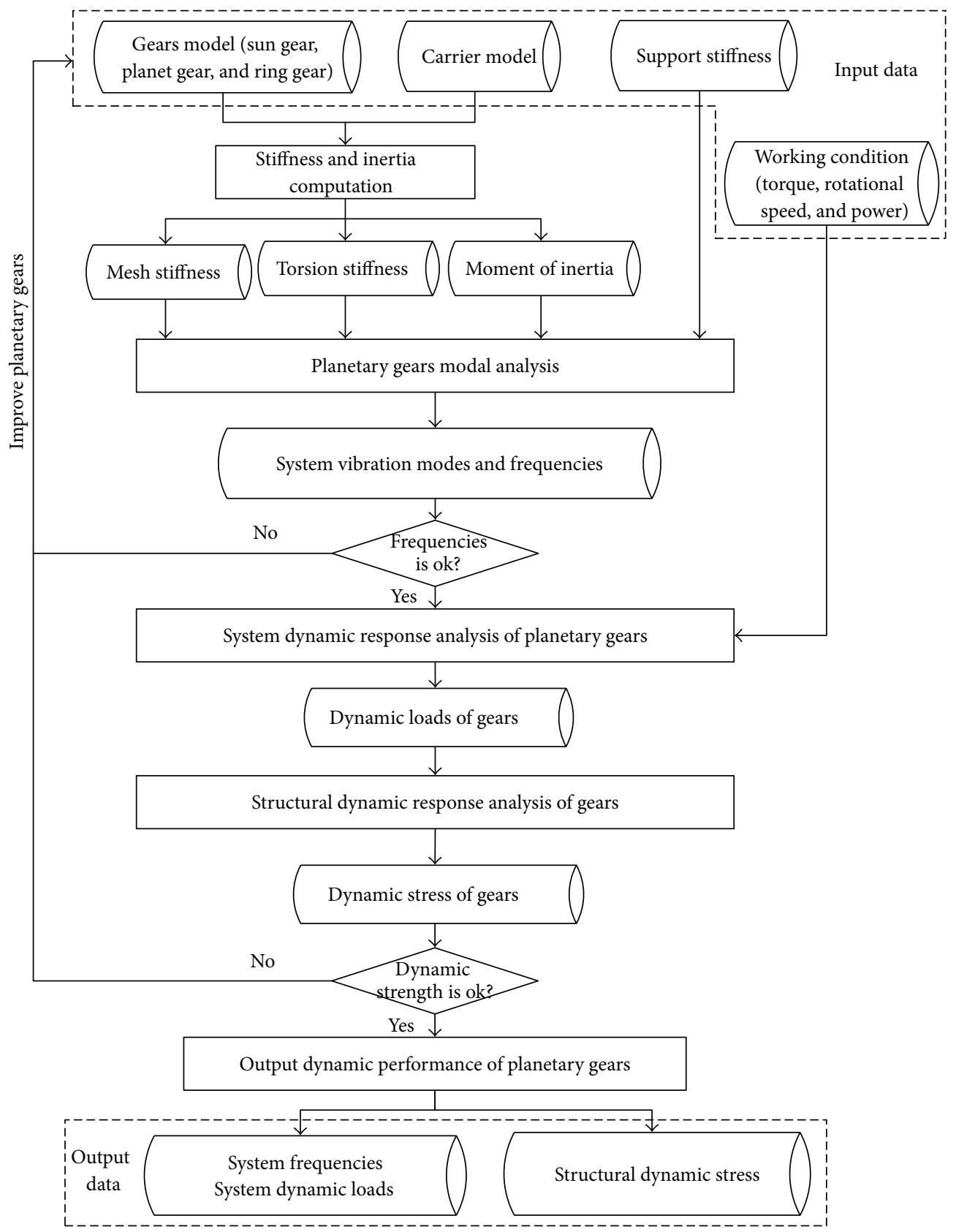

FIGURE 1: Flowchart of system-structure coupling dynamic analysis.

$$
\begin{aligned}
& \frac{J_{S}}{r_{S}^{2}} \ddot{u}_{S}+k_{S u} u_{S}+\sum_{i=1}^{N} k_{S i} \delta_{S P i}=\frac{T_{D}}{r_{s}}, \\
& m_{S} \ddot{x}_{S}+k_{S} x_{S}-\sum_{i=1}^{N} k_{S i} \delta_{S P i} \sin \varphi_{S i}=0, \\
& m_{S} \ddot{y}_{S}+k_{S} y_{S}+\sum_{i=1}^{N} k_{S i} \delta_{S P i} \cos \varphi_{S i}=0, \\
& \frac{J_{P}}{r_{P}^{2}} \ddot{u}_{P i}-k_{S i} \delta_{S P i}+k_{R i} \delta_{R P i}=0,
\end{aligned}
$$

$$
\begin{gathered}
m_{p}\left(\ddot{v}_{i}-2 \omega \dot{\xi}_{i}-\omega^{2} v_{i}-\dot{\omega} \xi_{i}\right)+k_{S i} \delta_{S P i} \sin \alpha \\
-k_{R i} \delta_{R P i} \sin \alpha+k_{P} \delta_{P i x}=0, \\
m_{p}\left(\ddot{\xi}_{i}+2 \omega \dot{v}_{i}-\omega^{2} \xi_{i}+\dot{\omega} v_{i}\right)+k_{S i} \delta_{S P i} \cos \alpha \\
-k_{R i} \delta_{R P i} \cos \alpha+k_{P} \delta_{P i y}=0, \\
\left(\frac{J_{C}}{r_{C}^{2}}+N m_{P}\right) \ddot{u}_{C}+k_{C u} u_{C}-\sum_{i=1}^{N} k_{S i} \delta_{S P i} \cos \alpha
\end{gathered}
$$




$$
\begin{aligned}
& +\sum_{i=1}^{N} k_{R i} \delta_{R P i} \cos \alpha=-\frac{T_{L}}{r_{C}} \\
& m_{C} \ddot{x}_{C}+k_{C x} x_{C}-\sum_{i=1}^{N} k_{P}\left(\delta_{P i x} \cos \varphi_{i}-\delta_{P i y} \sin \varphi_{i}\right)=0, \\
& m_{C} \ddot{y}_{C}+k_{C y} y_{C}-\sum_{i=1}^{N} k_{P}\left(\delta_{P i x} \cos \varphi_{i}+\delta_{P i y} \sin \varphi_{i}\right)=0, \\
& \frac{J_{R}}{r_{R}^{2}} \ddot{u}_{R}+k_{R u} u_{R}-\sum_{i=1}^{N} k_{R i} \delta_{R P i}=0, \\
& m_{R} \ddot{x}_{R}+k_{R x} x_{R}-\sum_{i=1}^{N} k_{R i} \delta_{R P i} \sin \varphi_{R i}=0, \\
& m_{R} \ddot{y}_{R}+k_{R y} y_{R}-\sum_{i=1}^{N} k_{R i} \delta_{R P i} \cos \varphi_{R i}=0, \\
& \delta_{S P i}=y_{S} \cos \varphi_{S i}-x_{S} \sin \varphi_{S i}+u_{S}-v_{i} \sin \alpha-\xi_{i} \cos \alpha \\
& +u_{P i}, \\
& \delta_{R P i}=y_{R} \cos \varphi_{R i}-x_{R} \sin \varphi_{R i}+u_{R}+v_{i} \sin \alpha-\xi_{i} \cos \alpha \\
& -u_{P i} \text {, } \\
& \delta_{P i x}=x_{C} \cos \varphi_{i}+y_{C} \sin \varphi_{i}-v_{i}, \\
& \delta_{\text {Piy }}=-x_{C} \sin \varphi_{i}+y_{C} \cos \varphi_{i}-\xi_{i}+u_{C}, \\
& \varphi_{i}=\frac{2 \pi(i-1)}{N}+\frac{\pi}{10}, \\
& \varphi_{S i}=\alpha-\varphi_{i}, \\
& \varphi_{R i}=\alpha+\varphi_{i},
\end{aligned}
$$$$
i=1,2, \ldots, N \text {. }
$$

Here, $r_{S}, r_{P i}(i=1,2, \cdots, N), r_{R}$ are the base radii; $r_{C}$ is the equivalent radius of the carrier; $\delta_{S P i}$ is the relative displacement between the sun gear and planet gear $i$ in the line of contact; $\delta_{R P i}$ is the relative displacement between the ring gear and planet gear $i$ in the line of contact; $\delta_{P i x}$ and $\delta_{P i y}$, respectively, are the relative displacements between the carrier and planet gear $i$ in the $x$ and $y$ direction in the local dynamic coordinate system of the planet gear.

Equation (1) is given in the matrix form as

$$
[M]\{\ddot{X}\}+[K(t)]\{X\}=\{P(t)\},
$$

where $[M]$ is the mass matrix, $[K(t)]$ is the time-varying stiffness matrix, $\{X\}$ is the displacement vector, and $\{P(t)\}$ is the load vector.

\subsection{Parameters Computation}

3.2.1. Time-Varying Mesh Stiffness of Gear Pair. Mesh stiffness of gear pair is varying with mesh position at all times. Timevarying mesh stiffness of gear pair should be considered in solving system dynamic equations. The total deformation $u(t)$ along the line of contact of a gear pair is generated when the gear pair meshes all the time. Therefore, the time-varying mesh stiffness of gear $K(t)$ pair is written as

$$
K(t)=\frac{T}{R_{b} u(t)},
$$

where $R_{b}$ is the base radius of the driving gear and $T$ is the input torque. The time-varying mesh stiffness is a periodic function in keeping with the mesh cycle of gear pair; one cycle of the mesh stiffness is shown in Figure 3.

3.2.2. Torsional Stiffness of the Shaft. Torsional stiffness of the shaft is computed by dividing shaft into a number of simple shafts which have a pair of interior and external diameter. The torsional stiffness of shaft $K_{s}$ is defined as

$$
\begin{aligned}
K_{s} & =\frac{G I_{p}}{L}, \\
I_{p} & =\frac{\pi\left(r_{2}^{4}-r_{1}^{4}\right)}{2},
\end{aligned}
$$

where $G$ is the shear modulus, $L$ is the length of the shaft, $r_{1}$ is the interior radius of the shaft, $r_{2}$ is the external radius of the shaft, and $I_{p}$ is the polar moment of inertia of the shaft.

3.2.3. Moment of Inertia of the Shaft. Computation method of the moment of inertia of the shaft is same as the torsional stiffness of the shaft. The moment of inertia of the shaft $J_{s}$ is defined as

$$
J_{s}=\frac{1}{2} \pi \rho\left(r_{2}^{4}-r_{1}^{4}\right) L,
$$

where $L$ is the length of the shaft, $r_{1}$ is the interior radius of the shaft, and $r_{2}$ is the external radius of the shaft.

3.3. Modal Analysis of Planetary Gears. Let the load vector $\{P(t)\}$ in (2) be a zero vector; then (2) can be used in modal analysis of planetary gears. According to the aforementioned computation method of time-varying mesh stiffness of gear pair, we can easily obtain the mean mesh stiffness to be applied in modal analysis. The mean mesh stiffness of the example planetary system is computed as $k_{S i}=1.088 \times$ $10^{9} \mathrm{~N} / \mathrm{m}$ and $k_{R i}=1.238 \times 10^{9} \mathrm{~N} / \mathrm{m}$. With assumption of $k_{\mathrm{Cu}}=6.0 \times 10^{8} \mathrm{~N} / \mathrm{m}, k_{S u}=5.0 \times 10^{8} \mathrm{~N} / \mathrm{m}, k_{R u}=$ $9.0 \times 10^{9} \mathrm{~N} / \mathrm{m}, k_{S}=2.0 \times 10^{8} \mathrm{~N} / \mathrm{m}, k_{P}=2.0 \times 10^{8} \mathrm{~N} / \mathrm{m}$, $k_{C x}=k_{C y}=3.0 \times 10^{8} \mathrm{~N} / \mathrm{m}$, and $k_{R x}=k_{R y}=9.0 \times 10^{8} \mathrm{~N} / \mathrm{m}$, the top twelve orders of natural frequencies of the example planetary gears can be calculated as Table 2 .

Two types of vibration modes exist in the modal analysis. First, distinct modes in which all components vibrate in rotational or translational mode are shown in Figure 4. The other type vibration mode is called degenerate mode in which only the planet gears vibrate and other components have no motion, as illustrated in Figure 5. 
TABLE 2: The top twelve orders of natural frequencies of the planetary gears.

\begin{tabular}{|c|c|c|c|c|c|c|c|c|c|c|c|c|}
\hline Order number & 1 & 2 & 3 & 4 & 5 & 6 & 7 & 8 & 9 & 10 & 11 & 12 \\
\hline Frequency, Hz & 1325 & 2106 & 2863 & 3594 & 3594 & 3594 & 3594 & 3989 & 4302 & 4766 & 5130 & 5288 \\
\hline
\end{tabular}

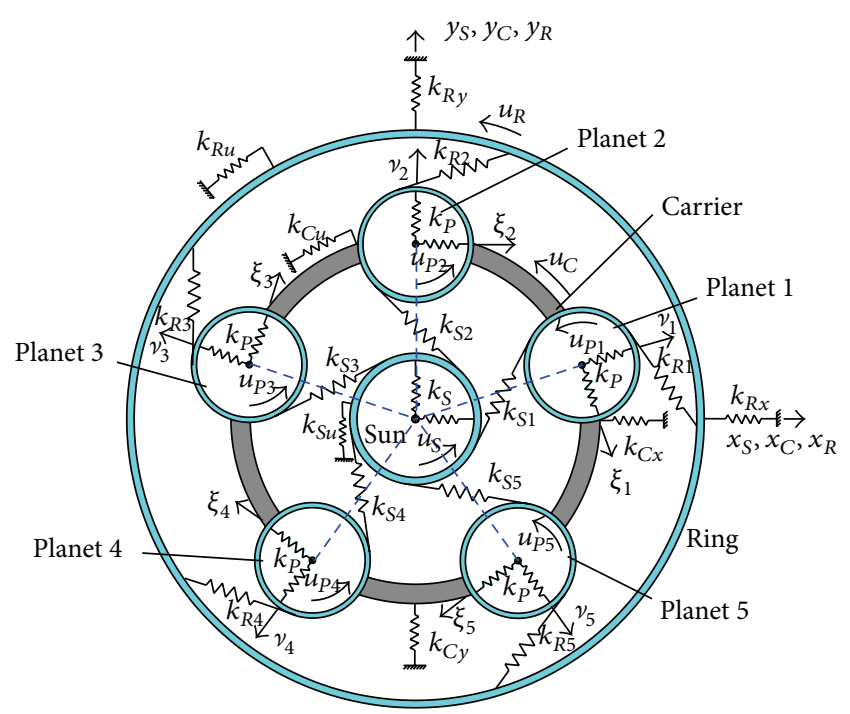

(a) Global system dynamic model

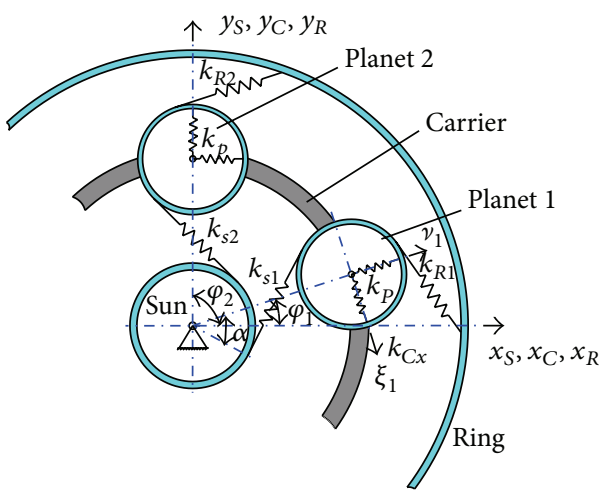

(b) Angles in dynamic model

Figure 2: System dynamic model of planetary gears.

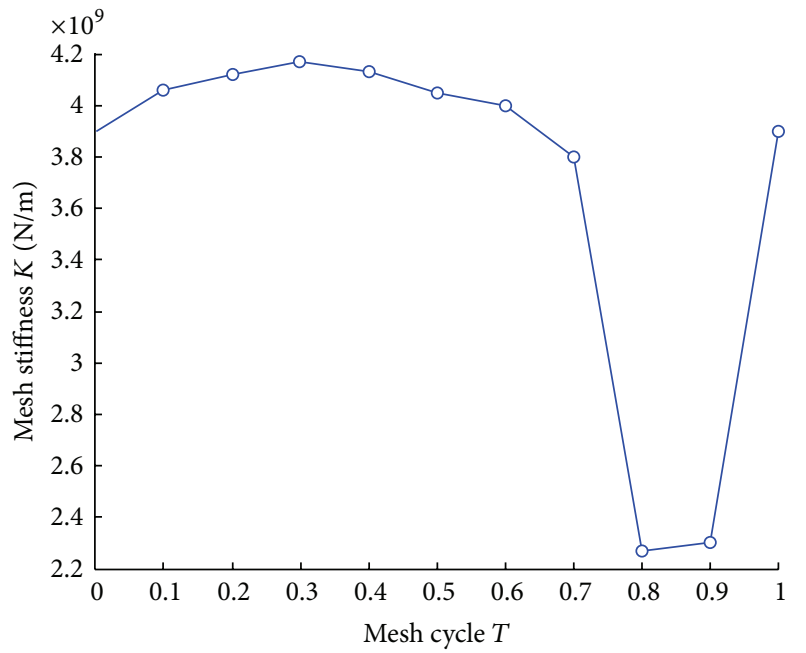

FIgURE 3: Time-varying mesh stiffness.

3.4. System Dynamic Response Analysis of Planetary Gears. The input power of the example planetary system is $1200 \mathrm{~kW}$, the input rotational speed is $1435 \mathrm{rpm}$, and the output rotational speed is $350 \mathrm{rpm}$. Assuming no power loss, we can obtain the values of input torque $\left(T_{D}\right)$ and output torque $\left(T_{L}\right)$ according to (9) as $T_{D}=7985 \mathrm{~N} \cdot \mathrm{m}$ and $T_{L}=32739 \mathrm{~N} \cdot \mathrm{m}$ :

$$
T=9549 \frac{P}{n}
$$

The exact values of the load vector $\{P(t)\}$ are then calculated based on (2). By introducing Fourier series, the dynamic meshing loads and rotational displacements on the gears of the planetary gears are obtained. Figure 6(a) plots the dynamic force between the sun gear and the planet gears, and Figure 6(b) plots the dynamic force between the planet gears and the ring gear. Figure 7 presents the dynamic rotational displacements of the sun gear, the planet gears, and the ring gear of the planetary gears.

\section{Structure Dynamic Analysis Based on FEM under Dynamic Loads}

4.1. Principle of Structure Dynamic Analysis Based on FEM. The principle of structure dynamic analysis based on FEM is described as follows.

4.1.1. Structure Dynamic Equation. The first step of structure dynamic analysis based on FEM is similar to static analysis, which is to separate structure into many elements. And then the whole finite element (FE) dynamic equations of separated structure are built on basis of single element dynamic equation. Hamilton's principle is used as a variation principle to build the whole FE equation.

Hamilton's Principle. Among all the possible time-variant displacement forms that satisfy the compatibility condition, constraint condition, motion boundary condition, and time 


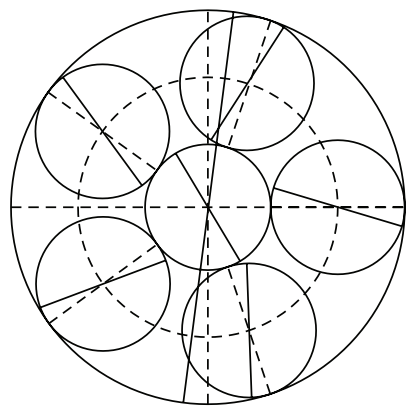

(a) $f=1325 \mathrm{~Hz}$

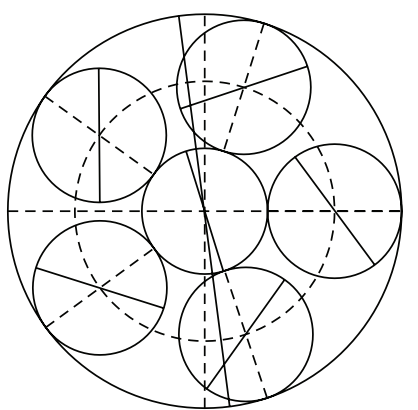

(e) $f=4302 \mathrm{~Hz}$

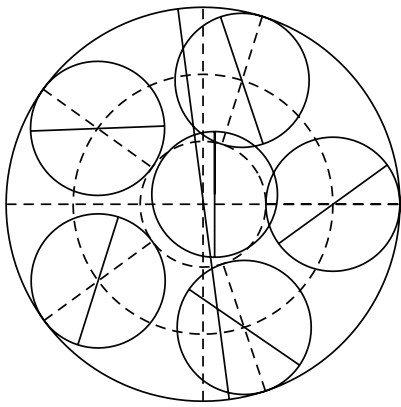

(b) $f=2106 \mathrm{~Hz}$

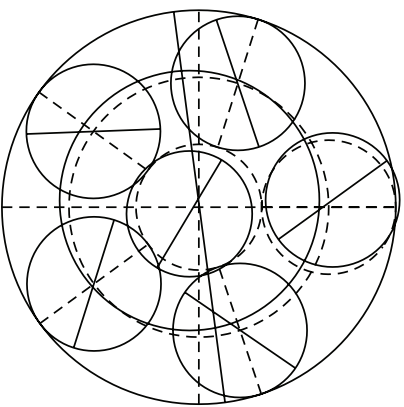

(f) $f=4766 \mathrm{~Hz}$

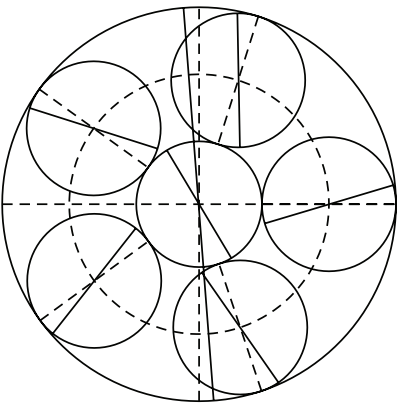

(c) $f=2863 \mathrm{~Hz}$

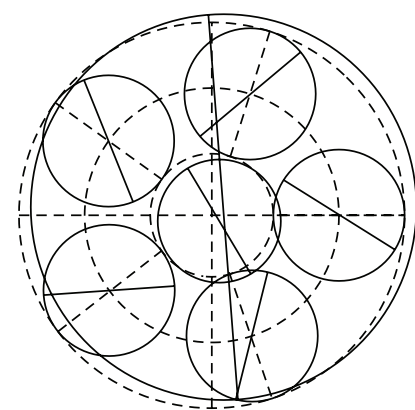

(g) $f=5130 \mathrm{~Hz}$

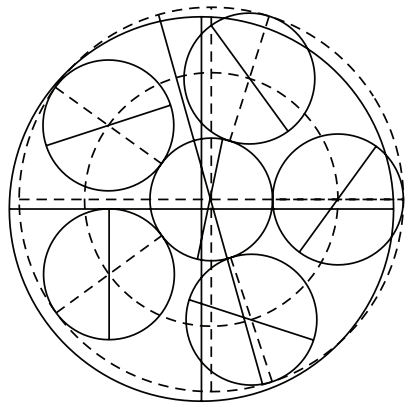

(d) $f=3989 \mathrm{~Hz}$

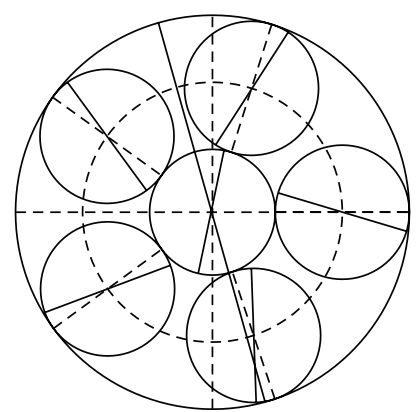

(h) $f=5288 \mathrm{~Hz}$

FIGURE 4: Distinct mode.
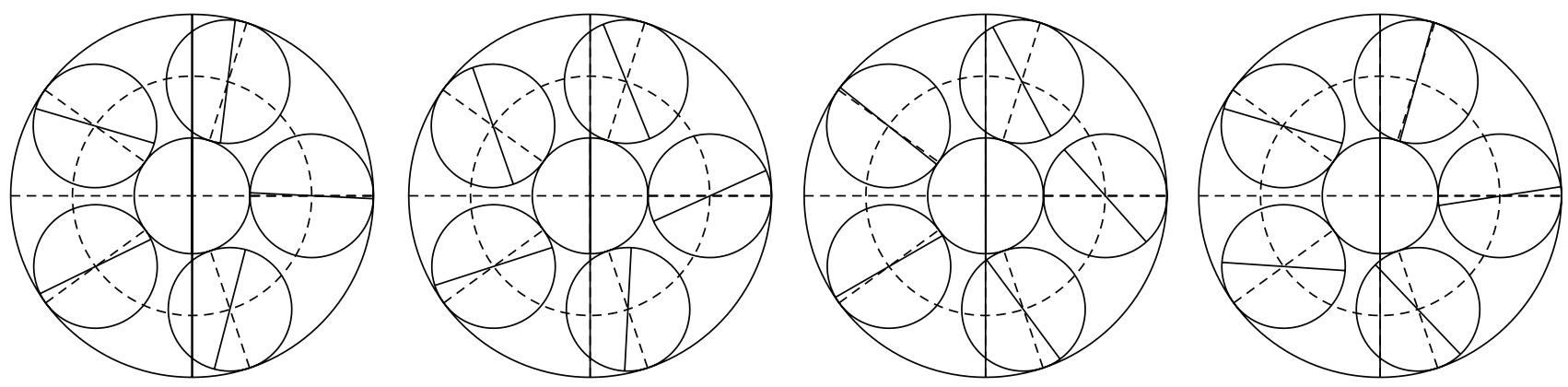

Figure 5: Degenerate mode.

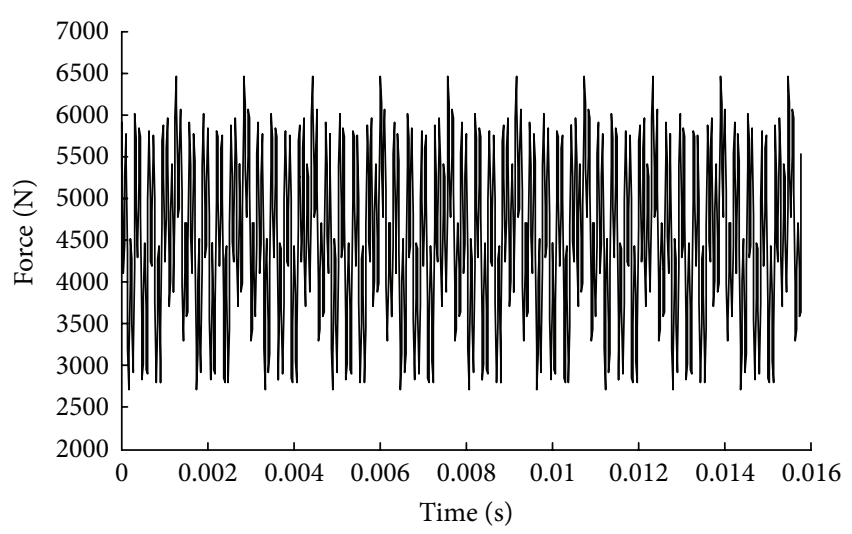

(a) Force between the sun gear and the planet gears

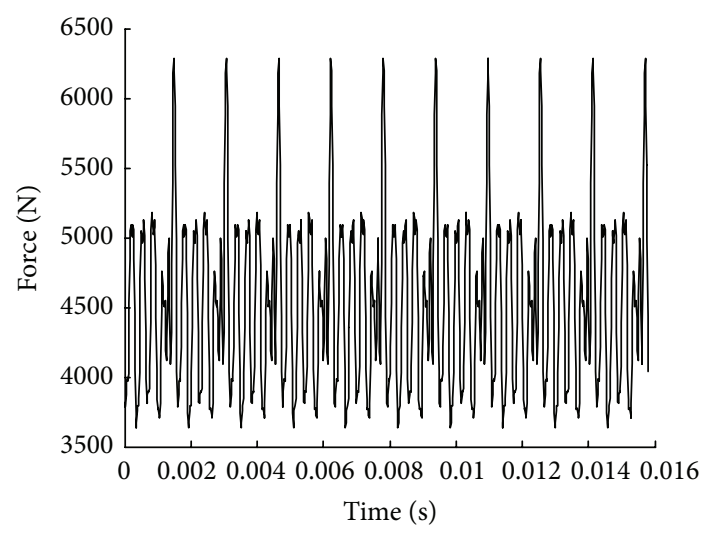

(b) Force between the planet gears and the ring gear

Figure 6: Dynamic meshing loads on the gears of the planetary gears. 


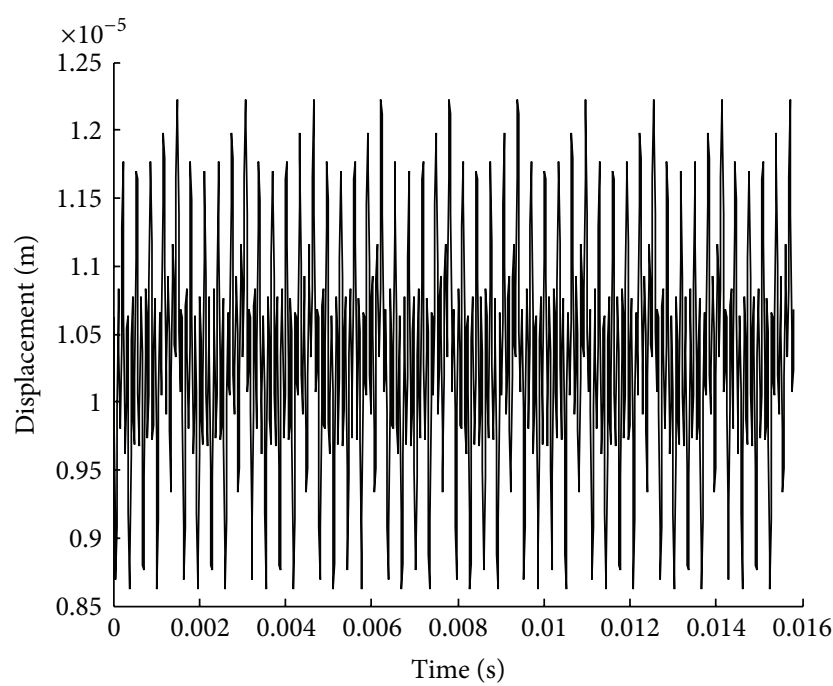

(a) The sun gear

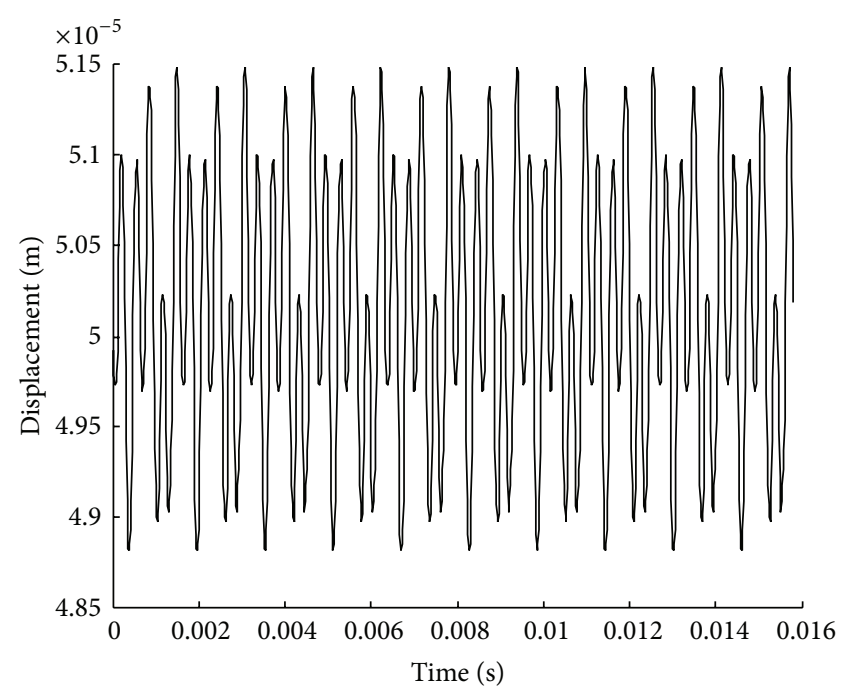

(b) The planet gears

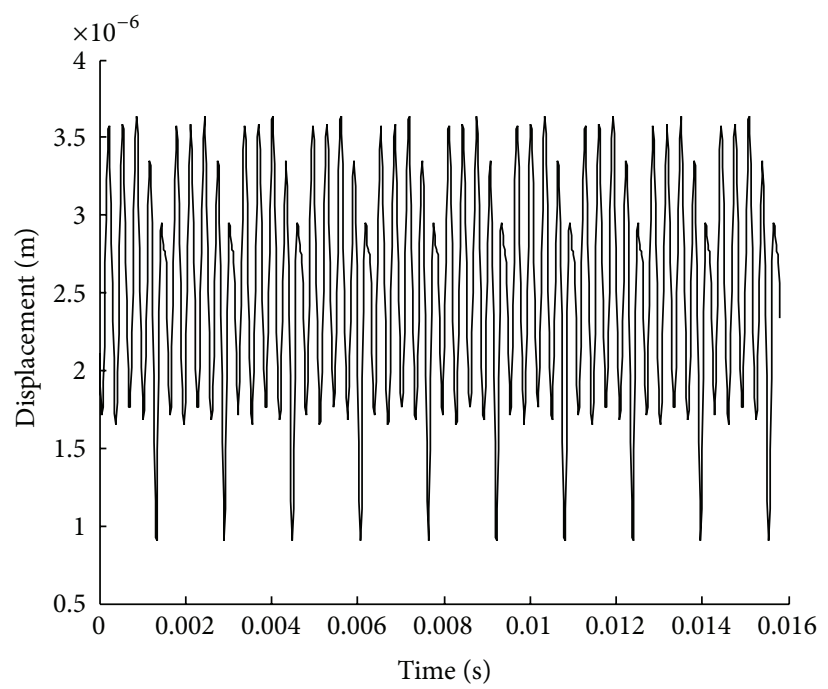

(c) The ring gear

FIGURE 7: Dynamic rotational displacements of the gears of the planetary gears.

condition, the variation form of real solution minimizes the Lagrange function [18].

Element dynamic equation is written as

$$
\left[M_{e}\right]\left\{\ddot{\delta}_{e}\right\}+\left[C_{e}\right]\left\{\dot{\delta}_{e}\right\}+\left[K_{e}\right]\left\{\delta_{e}\right\}=\left\{F_{e}(t)\right\},
$$

where $\left[M_{e}\right]$ is the element mass matrix, $\left[C_{e}\right]$ is the element damping matrix, $\left[K_{e}\right]$ is the element stiffness matrix, $\left\{F_{e}(t)\right\}$ is the node transient variant force vector, and $\left\{\delta_{e}\right\}$ is the node displacement vector.

Through assembling all the element dynamic equations (7), the whole FE dynamic equations of structure system are obtained as

$$
[M]\left\{\ddot{\delta}_{s}\right\}+[C]\left\{\dot{\delta}_{s}\right\}+[K]\left\{\delta_{s}\right\}=\left\{F_{s}(t)\right\},
$$

where $[M]$ is the system mass matrix, $[C]$ is the system damping matrix, $[K]$ is the system stiffness matrix, $\left\{F_{s}(t)\right\}$ is the transient variant force vector of all nodes, and $\left\{\delta_{s}\right\}$ is the displacement vector of all nodes.

4.1.2. Solution Method of Structure Dynamic Equation. The structure dynamic equations (8) are second-order differential equations. If there are no external excitation forces, namely, the right hand size $\left\{F_{s}(t)\right\}=\{0\}$ of $(8)$, nonzero solution of the equations can be obtained by means of free vibration analysis. The solution reflects the natural characteristics of the structure, namely, natural frequencies and vibration modes, which are called eigenvalues and eigenvectors. In fact, damping has little influence to natural frequencies and vibration modes of structure; therefore it is ignored in natural characteristics analysis. Equation (8) is simplified as

$$
[M]\left\{\ddot{\delta}_{s}\right\}+[K]\left\{\delta_{s}\right\}=\{0\} .
$$




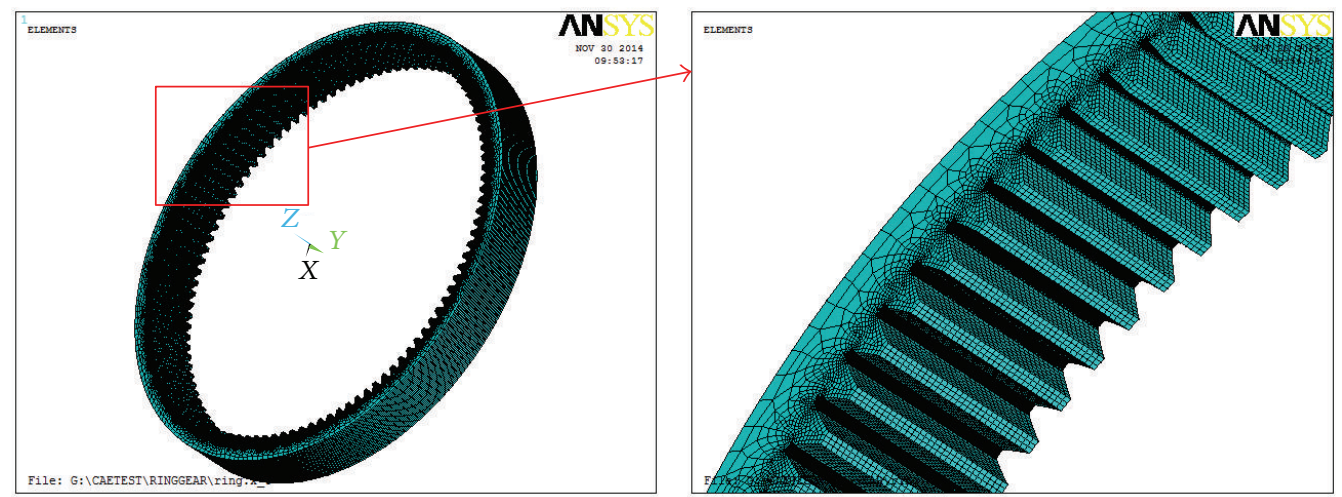

FIGURE 8: The finite element model of the ring gear. tion,

Assuming that the structure has simple harmonic vibra-

$$
\begin{aligned}
& \left\{\delta_{s}\right\}=\{\phi\} \sin (\omega t+\theta), \\
& \left\{\ddot{\delta}_{s}\right\}=-\omega^{2}\{\phi\} \sin (\omega t+\theta),
\end{aligned}
$$

where $\omega$ is the circular frequency, $\theta$ is the starting phase angle, $t$ is the time variable, and $\{\phi\}$ is the displacement amplitude unrelated with $t$.

Equation (9) in conjunction with (10) yields an equation as

$$
[K]\{\phi\}=\lambda[M]\{\phi\},
$$

where $\lambda=\omega^{2}$. Such equation is a generalized eigenvalue solving problem of structure dynamics. The kernel of the problem is to obtain $\lambda$ and nonzero solution $\{\phi\}$. Obviously, the equation solution is only determined by the stiffness matrix $[K]$ and the mass matrix $[M]$ of the structure. $\lambda$ denotes the eigenvalue, and the corresponding vibration mode is the eigenvector. In three dimensional dynamics, since both mass matrix $[M]$ and stiffness matrix $[K]$ are symmetric positive definite matrices, all eigenvalues are nonnegative real numbers.

Modal coordinates should be transformed when the structure dynamic equations (8) are solved; that is, dynamic equations in the original physical space are transformed into the modal space. The transformation method adopted is mode superposition method. The front $p$ orders of fundamental modes $\varphi=\left[\varphi_{1}, \varphi_{2}, \ldots, \varphi_{p}\right]$ comprise coordinate transformation matrix. So the order number of system model is decreased. Equation (8) is simplified as $p$ second-order differential equations which are independent to each other. The displacement response of the structure is computed according to these equations. Then dynamic stress components $\sigma_{x}(t), \sigma_{y}(t), \sigma_{z}(t), \tau_{x y}(t), \tau_{y z}(t)$, and $\tau_{z x}(t)$ are calculated. Equivalent stress $\bar{\sigma}(t)$ according to Von Mises yield criterion is defined as

$$
\begin{aligned}
\bar{\sigma}(t)= & \sqrt{3} \\
& \cdot\left(\frac{1}{2}\left(\sigma_{x}^{\prime 2}+\sigma_{y}^{\prime 2}+\sigma_{z}^{\prime 2}\right)+\tau_{x y}^{2}+\tau_{y z}^{2}+\tau_{z x}^{2}\right)^{1 / 2}, \\
\sigma_{x}^{\prime}= & \sigma_{x}-\frac{1}{3}\left(\sigma_{x}+\sigma_{y}+\sigma_{z}\right), \\
\sigma_{y}^{\prime}= & \sigma_{y}-\frac{1}{3}\left(\sigma_{x}+\sigma_{y}+\sigma_{z}\right), \\
\sigma_{z}^{\prime}= & \sigma_{z}-\frac{1}{3}\left(\sigma_{x}+\sigma_{y}+\sigma_{z}\right) .
\end{aligned}
$$

4.2. Structure Dynamic Analysis of Gears. Structure dynamic analysis is a general method to explore the vibration characteristics and to verify the dynamic structure strength of all gears. By using the commercial software ANSYS, the structure dynamic analysis of the planetary gears is carried out. In order to illustrate the detail method, now we take the ring gear as an example, whose FE model and analysis results are described below.

4.2.1. Ring Gear FE Model. The finite element model of the ring gear is built by using ANSYS and is shown in Figure 8. The model consisted of 693222 elements and 760151 nodes. The material of the ring gear is one kind of killed steel, whose elastic modulus $E$ is $210 \mathrm{GPa}$, poisson ratio $\mu$ is 0.3 , and density $\rho$ is $7800 \mathrm{~kg} / \mathrm{m}^{3}$. The constraint condition of the FE model is that the displacements of all the exterior ring nodes are zeros. Denser grids in gear roots ensure high computation accuracy for analyzing dynamic response of the ring gear.

4.2.2. Dynamic Response Analysis of Ring Gear. One period of dynamic loads is applied on five gear teeth engaged with five planet gears by forty load steps in one mesh period $(0.0016$ seconds), as displayed in Figure 9.

At every load step, the ring gear transient dynamic response is solved, and then the entire dynamic stresses and 


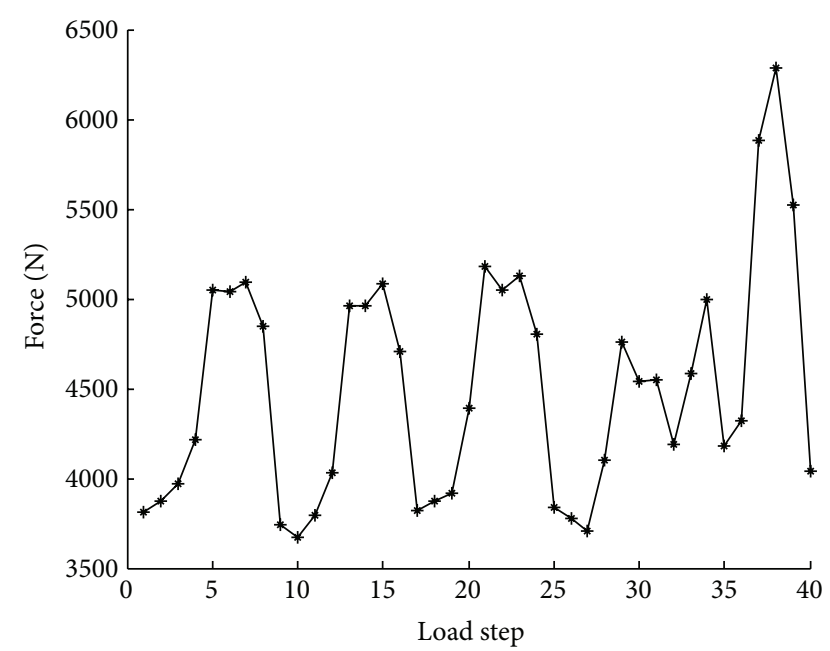

Figure 9: Dynamic loads applied on gear teeth.

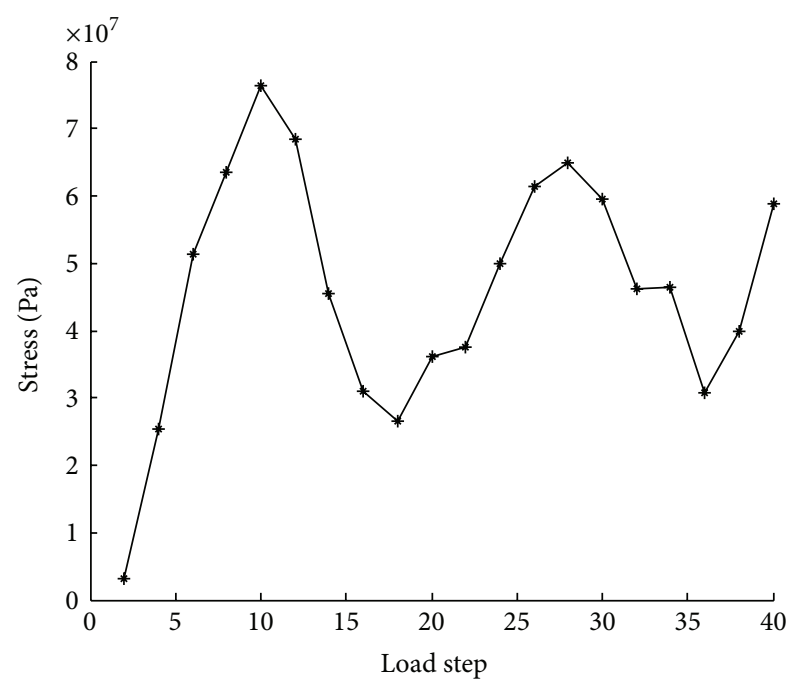

Figure 10: Dynamic maximum stresses of the ring gear.

displacements of all nodes can be obtained in this load period. Figure 10 shows the dynamic maximum stresses of the ring gear at every even load step. Among these twenty stresses, the maximum stress is $76.45 \mathrm{MPa}$, which is less than the critical strength $(121 \mathrm{MPa})$ of the material. The result indicates the ring gear is safe to run in such working situation. If the maximum stress is higher than the critical strength, the ring gear has to be redesigned and its dynamic response recomputed for evaluating its safety. Finally, after analyzing and redesigning all parts of planetary gears by using the proposed method, an optimal scheme of the planetary gears is achieved.

\section{Conclusions}

In this paper, a novel general method of system-structure coupling dynamic analysis is presented, which is used to analyze planetary gears dynamic vibration when the influences of system parameters and structure dimensions are considered at the same time. The lumped parameter system dynamic model of the planetary gears is built, and the structure dynamic analysis method is presented in detail by introducing the FEM method. The coupling method of the two dynamic models is completed through the dynamic loads on gear teeth obtained from system dynamics. Compared to existing models according to the results, the proposed coupling dynamic analysis method has some advantages below:

(1) Since all components are simplified as rigid bodies in lumped parameter model, structure flexibility is considered in the proposed model; so it demonstrates more accurate than lumped parameter model in analyzing gears structure dynamics.

(2) The proposed analysis method is easier to develop the model through coupling system dynamics and structure dynamics than distributed mass model and rigid-flexible coupling model. Moreover, it can save computer time than these models in calculating the dynamic equations of the planetary gears.

The proposed coupling dynamic analysis method is a convenient and efficient approach to analyze full-scale dynamic characteristics of planetary gears, which considers system vibration modes, natural frequencies, dynamic loads on gears, structure dynamic displacements, and dynamic stresses. The analysis results can be used as the direct evidence to estimate dynamic performances of the planetary gears.

\section{Conflict of Interests}

The authors declare that they have no conflict of interests regarding the publication of this paper.

\section{Acknowledgments}

This research is supported by the Northwestern Polytechnical University basic research foundation Project (no. JC201209) and the 111 Project (no. B13044).

\section{References}

[1] J. Lin and R. G. Parker, "Analytical characterization of the unique properties of planetary gear free vibration," Journal of Vibration and Acoustics, Transactions of the ASME, vol. 121, no. 3, pp. 316-321, 1999.

[2] T. L. Krantz, "Mechanical systems technology branch research summary 1985-1992," NASA Technical Memorandum 106329, 1993.

[3] A. Kahraman, "Natural modes of planetary gear trains," Journal of Sound and Vibration, vol. 173, no. 1, pp. 125-130, 1994.

[4] S. Y. Wang, C. Zhang, Y. M. Song, and T. Q. Yang, "Natural mode analysis of planetary gear trains," China Mechanical Engineering, vol. 16, no. 16, pp. 1461-1465, 2005.

[5] R. G. Parker, V. Agashe, and S. M. Vijayakar, "Dynamic response of a planetary gear system using a finite element/contact 
mechanics model," Journal of Mechanical Design, vol. 122, no. 3, pp. 304-310, 2000.

[6] A. Saada and P. Velex, "Extended model for the analysis of the dynamic behavior of planetary trains," Transactions of the ASME-Journal of Mechanical Design, vol. 117, no. 2, pp. 241247, 1995.

[7] T. Yang, Y. Song, C. Zhang, and S. Wang, "Property analysis of free vibration of helical planetary gear trains," Chinese Journal of Mechanical Engineering, vol. 41, no. 7, pp. 50-55, 2005.

[8] V. K. Ambarisha and R. G. Parker, "Nonlinear dynamics of planetary gears using analytical and finite element models," Journal of Sound and Vibration, vol. 302, no. 3, pp. 577-595, 2007.

[9] A. Singh, "Application of a system level model to study the planetary load sharing behavior," Journal of Mechanical Design, vol. 127, no. 3, pp. 469-476, 2005.

[10] X. Wu and R. G. Parker, "Modal properties of planetary gears with an elastic continuum ring gear," Transactions ASMEJournal of Applied Mechanics, vol. 75, no. 3, Article ID 031014, 2008.

[11] R. G. Parker and X. Wu, "Vibration modes of planetary gears with unequally spaced planets and an elastic ring gear," Journal of Sound and Vibration, vol. 329, no. 11, pp. 2265-2275, 2010.

[12] V. Abousleiman and P. Velex, "A hybrid 3D finite element/lumped parameter model for quasi-static and dynamic analyses of planetary/epicyclic gear sets," Mechanism and Machine Theory, vol. 41, no. 6, pp. 725-748, 2006.

[13] T. M. Ericson and R. G. Parker, "Natural frequency clusters in planetary gear vibration," Journal of Vibration and Acoustics, Transactions of the ASME, vol. 135, no. 6, Article ID 061002, 2013.

[14] R. G. Parker and X. Wu, "Parametric instability of planetary gears having elastic continuum ring gears," Journal of Vibration and Acoustics, vol. 134, no. 4, Article ID 041011, 2012.

[15] C.-J. Bahk and R. G. Parker, "Analytical solution for the nonlinear dynamics of planetary gears," Journal of Computational and Nonlinear Dynamics, vol. 6, no. 2, Article ID 021007, 2011.

[16] V. K. Ambarisha and R. G. Parker, "Suppression of planet mode response in planetary gear dynamics through mesh phasing," Journal of Vibration and Acoustics, vol. 128, no. 2, pp. 133-142, 2006.

[17] Y. Guo and R. G. Parker, "Purely rotational model and vibration modes of compound planetary gears," Mechanism and Machine Theory, vol. 45, no. 3, pp. 365-377, 2010.

[18] M. Boscolo and J. R. Banerjee, "Dynamic stiffness laminate elements for exact modal analysis of aeronautical structures," in Proceedings of the 53rd AIAA/ASME/ASCE/AHS/ASC Structures, Structural Dynamics and Materials Conference, Honolulu, Hawaii, USA, April 2012. 


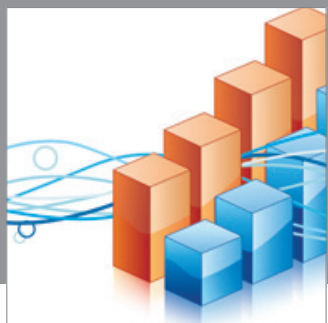

Advances in

Operations Research

mansans

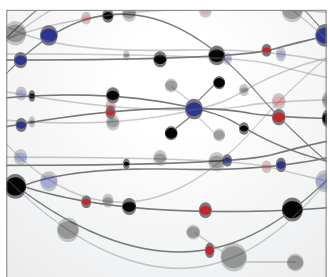

The Scientific World Journal
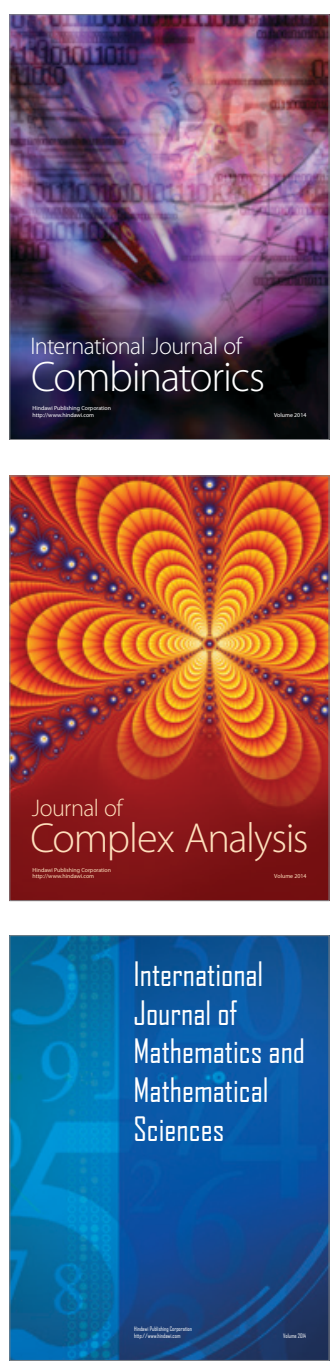
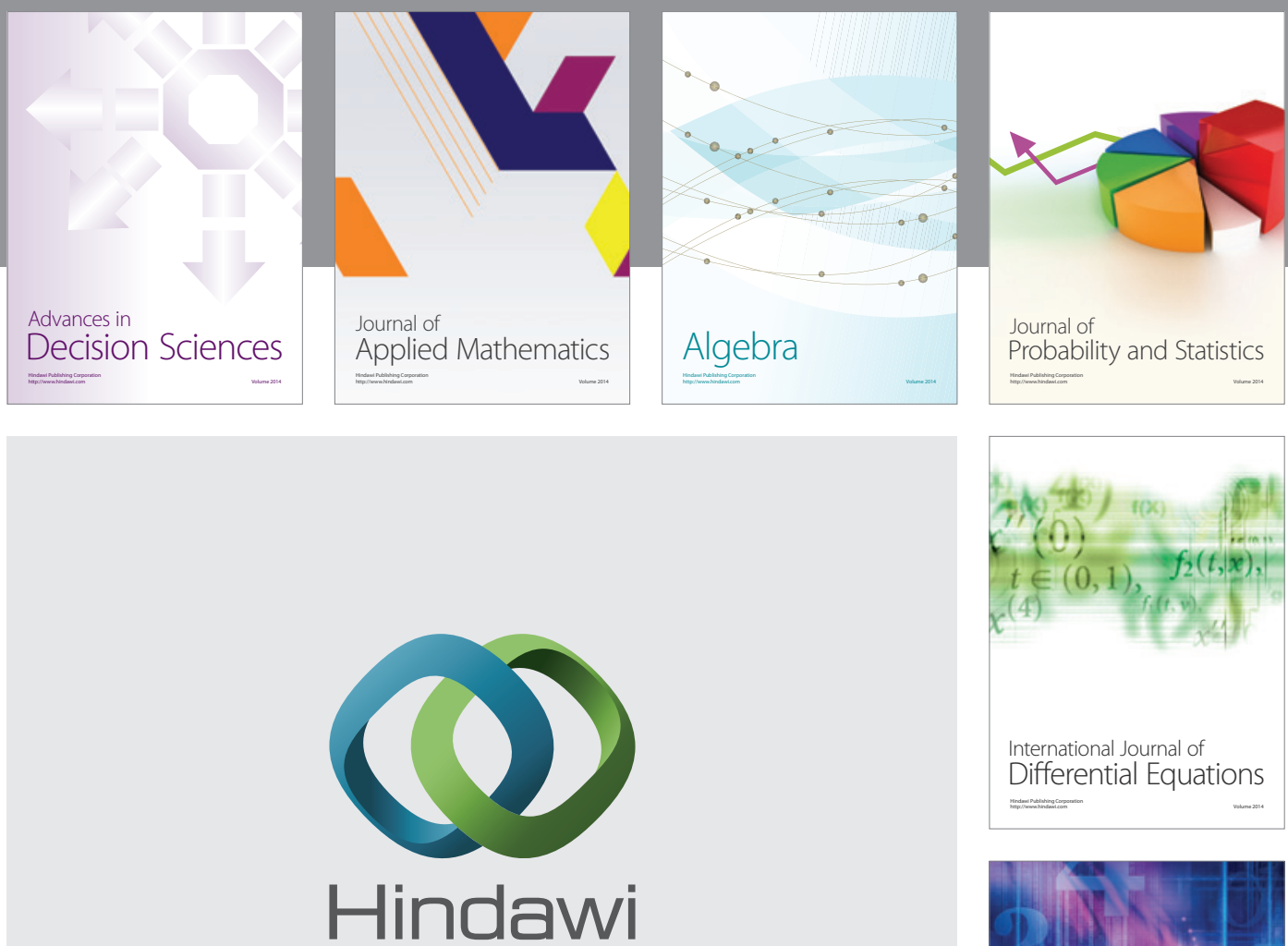

Submit your manuscripts at http://www.hindawi.com
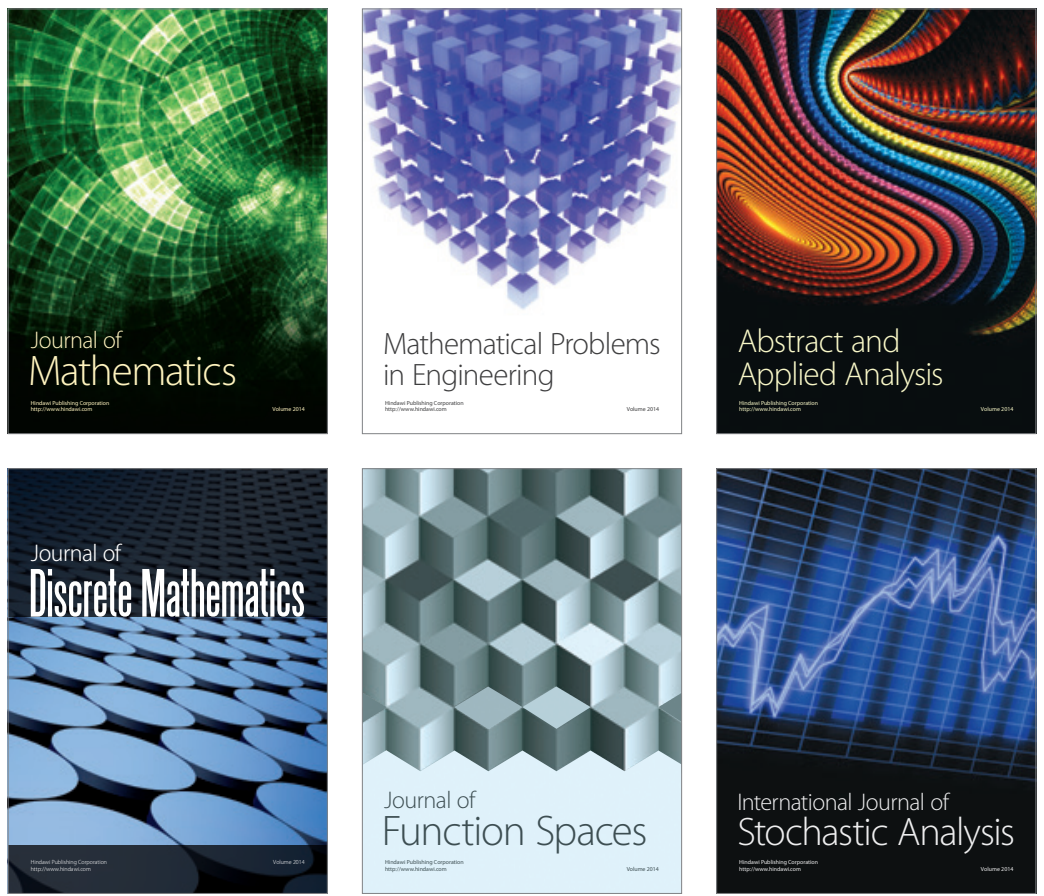

Journal of

Function Spaces

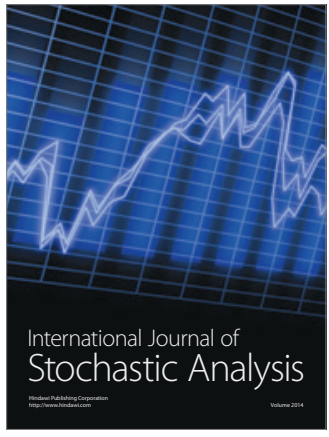

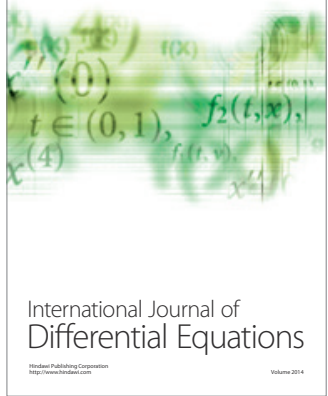
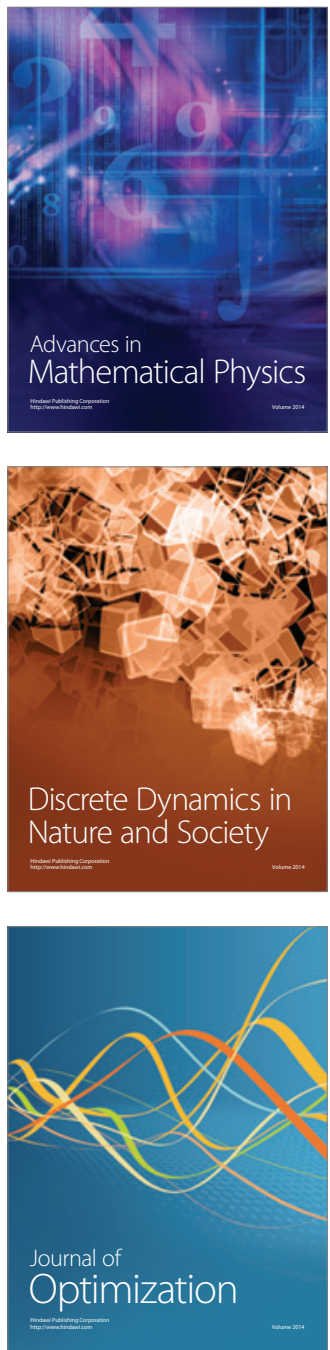